\title{
TESTING COSMOLOGICAL MODELS WITH CLUSTERS OF GALAXIES
}

\author{
S. GOTTLÖBER AND J. RETZLAFF \\ Astrophysical Institute Potsdam \\ An der Sternwarte 16, D-14482 Potsdam \\ AND
}

A. KLYPIN

Department of Astronomy, New Mexico State University Las Cruces, NM 88001, USA

\section{Introduction}

Currently little is known about the mass distribution on intermediate scales between those probed by deep redshift surveys of galaxies and those probed by COBE. Catalogs of galaxy clusters reach depths of several hundred megaparsecs, and, thus, are very useful for those scales. Only the Las Campanas Redshift Survey (LCRS) is comparable with that depth. However, the LCRS samples only narrow slices whereas cluster catalogs cover a large fraction of the sky. Clusters seems to be the most suitable objects to fill the gap between scales probed by COBE and the galaxy samples. Moreover, clusters are advantageous over galaxies as probes of the matter distribution in the Universe because our understanding of its formation and evolution is better established than it is for galaxies. Clusters are high peaks (mass scale $M \simeq 10^{15} M_{\odot}$ ) in the density field, which have collapsed relatively recently. Because of that, it is easy to identify clusters in numerical simulations. But the number of clusters is much smaller than the number of galaxies, which makes the statistics of clusters noisier. Nevertheless, clusters are exceptionally useful objects for the investigation of the matter distribution on scales well above $100 h^{-1} \mathrm{Mpc}$. Thus, it is worth to apply different statistical tests to these objects.

We have performed numerical simulations for a number of different cosmological models, all of them with a substantial part of cold dark matter. Using the simulations we have constructed mock samples of clusters which 
have the same boundaries and selection creteria as the original observational sample. We discuss the power spectrum of the cluster sample and compare it with those of the mock samples and we discuss briefly other statistics.

\section{Cosmological models}

We consider seven different cosmological models with cold dark matter: the standard cold dark matter (SCDM) model, a low density open cosmological model (OCDM), a model with a tilted primordial spectrum (TCDM), spatially flat models with a cosmological constant $(\Lambda \mathrm{CDM})$ and with a mixture of cold and hot dark matter (CHDM) with two equal mass neutrinos (Primack et al. 1995), a model with a decaying massive neutrino $(\tau \mathrm{CDM})$ proposed by Efstathiou et al. (1992) and a broken scale invariant model (BSI) (Gottlöber et al. 1994). These models and their parameters are summarized in the following table:

$\begin{array}{lllll}\text { SCDM } & h & \Omega & & \\ \text { OCDM } & h & \Omega & & \\ \text { TCDM } & h & \Omega & n & \\ \Lambda \text { CDM } & h & \Omega_{C D M} & \Omega_{\Lambda} & \\ \text { CHDM } & h & \Omega_{C D M} & \Omega_{H D M} & n_{\nu} \\ \tau \text { CDM } & h & \Omega & m_{\tau} & t_{\tau} \\ \text { BSI } & h & \Omega & k_{b r} & \Delta\end{array}$

The simplest models are described by two free parameters, the Hubble constant $h$ and the total matter density which is assumed to be $\Omega=1$ (Einstein-deSitter model). The TCDM and $\Lambda$ CDM models are described by one additional free parameter (the power index $n$ or the value of the cosmological constant, i.e. $\Omega_{\Lambda}$, whereas $\Omega_{\text {matter }}+\Omega_{\Lambda}=1$. The $\tau \mathrm{CDM}$ and BSI models have two additional free parameters. The mass and life time of the massive particle are related to the scale where the spectrum changes and the amount of change relative to the SCDM model. The spectrum is quite similar to the BSI spectrum (see Fig. 1), where the scale of the break in the spectrum $k_{b r}$ and the relative height of the step $\Delta$ are related to properties of the fields in the underlying double inflationary scenario (Gottlöber et al. 1991). Note that all of these models are additionally characterized by the small amount of baryonic matter $\Omega_{b a r}$, which however does not influence the power spectrum on cluster scales.

It is clear that the models fit the data better with increasing number of parameters. Therefore, with three additional parameters also open tilted 


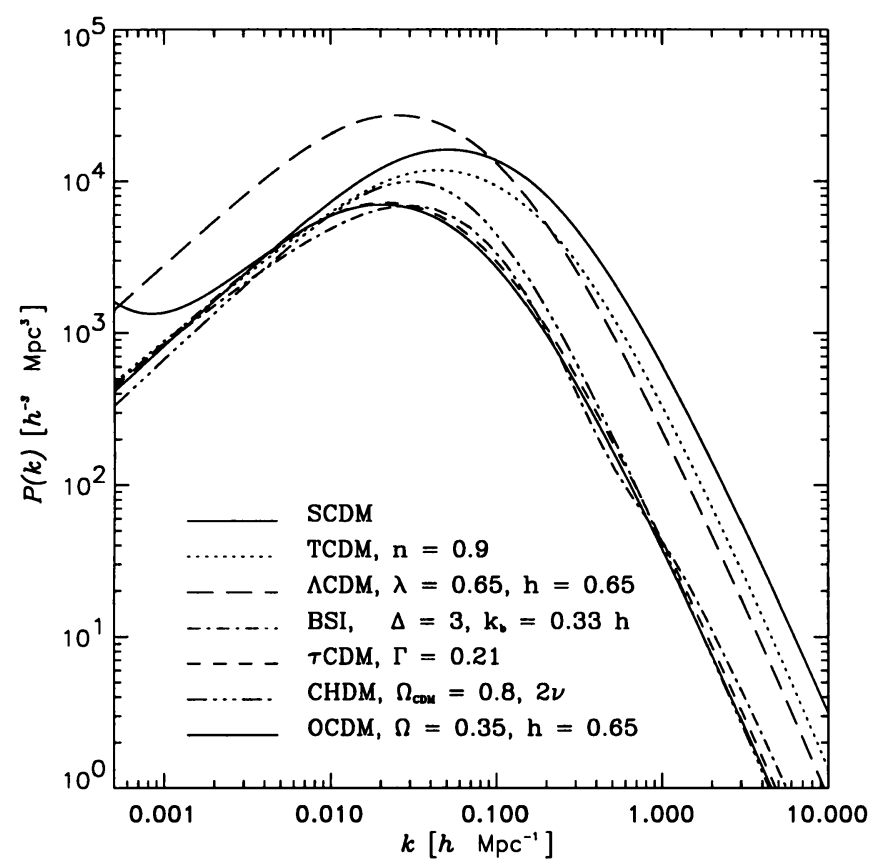

Figure 1. The power spectrum of different cosmological models in COBE-normalisation

models (OTCDM) or models with a cosmological constant and a tilted primordial spectrum $(\Lambda \mathrm{TCDM})$ or an additional hot dark matter component $(\Lambda \mathrm{CHDM})$ have been discussed in the literature.

\section{Clusters of galaxies - observation vs. simulation}

We consider an extended redshift sample of Abell and ACO clusters with richness $R \geq 0$ (Abell 1958; Abell, Corwin \& Olowin 1989). This sample was compiled by Plionis \& Valdarnini (1991) (for details see also Borgani et al. 1996; Plionis \& Valdarnini 1995). The galactic absorption is modelled according to the standard cosecant dependence on the galactic latitude and the cluster-redshift selection function is determined by fitting the cluster density as a function of redshift. In order to limit the effects of galactic absorption and errors due to the low density on large distances only clusters with a latitude $\geq 30^{\circ}$ and a distance $\leq 240 h^{-1} \mathrm{Mpc}$ are taken into account. The resulting catalog of $417 \mathrm{Abell} / \mathrm{ACO}$ clusters is approximately volume limited.

The power spectrum of this sample of Abell/ACO clusters has been determined by Retzlaff et al. (1997). In the calculation of the power spectrum 
the main attention has to be focused on the selection effects. Accounting for the geometry of the sample, and, more subtle, proper correction for the selection in redshift and galactic latitude are important. The power spectrum of the Abell/ACO clusters (stars) is shown in Fig. 2. For $k>0.05 h$ $\mathrm{Mpc}^{-1}$ the cluster power spectrum is well approximated by a power law, $P(k) \propto k^{n}$ with $n \simeq-1.9$, while it changes sharply to a positive slope at smaller wavenumbers.

Recently, the cluster correlation function has been estimated for a sample of 1304 Abell/ACO clusters of galaxies (Einasto et al. 1997a). The Fourier transform of the correlation function gives the power spectrum, which shows a pronounced peak at $100 h^{-1} \mathrm{Mpc}$ scale (Einasto et al. 1997b, see also Einasto, this volume). The parent cluster catalog - the Abell/ACO catalog - is the same for both determinations of the power spectrum. While the selection of richness class $R \geq 0$ clusters within the angular boundaries dictated by galactic absorption is also identical for our sample and that used by Einasto et al., the redshift samples are very different. The sample used by Einasto et al. extends to a depth of $340 h^{-1} \mathrm{Mpc}$ with a high fraction of estimated cluster redshifts (33\%). For our sample this fraction is just $4 \%$. Besides the difference in the observational data, we use different methods for estimating the power spectrum. Finding the same $P(k)$ slope of $n \approx-1.9$ with both methods clearly demonstrates the robustness of this result. The maximum of $P(k)$ is situated at the same scale, though the sharp peak which was found by Einasto et al. turns out to be less significant from our analysis. Recently, Gaztañaga and Baugh (1997) found a steep slope for the real space power spectrum of APM galaxies in the same range as the steep slope of the cluster power spectrum.

To compare the observed power spectrum with those predicted by different cosmological models we have performed a series of numerical simulations from which we have extracted mock catalogs with the same properties as the observational catalog. We normalized the power spectra according to the two year COBE measurement following Górski et al. (1994) and Stompor, Górski \& Banday (1995). However, we checked also that the slightly lower four year normalisation does not change the result. We used a standard PM $N$-body code with $N_{\mathrm{p}}=256^{3}$ particles and $N_{\mathrm{g}}=512^{3}$ grid cells in a simulation box of $L=500 h^{-1} \mathrm{Mpc}$ comoving length. This provides a dynamical range of about $0.9 h^{-1} \mathrm{Mpc}$ (one cell). We made simulations for four random realizations for SCDM, three realizations for $\Lambda \mathrm{CDM}$, and one realization for each of the other models. Thus, we were able to assess the statistical variance for SCDM and $\Lambda$ CDM while assuming a similar behaviour for the other models.

We use two different methods for determining the position of galaxy clusters in the simulation. The first is based on the friend-of-friend (FOF) 
algorithm and the second is based on the peak-of-density algorithm. We find the centers of groups of particles using the FOF method or the location of peaks in the density field. In the next step we center spheres with the Abell radius $1.5 h^{-1} \mathrm{Mpc}$ around these points and determine the centers of mass of the particles inside these spheres. After few iterations the centers of mass converge. We find that both methods identify the same clusters in almost all cases. We use the same linking length of 0.2 (in units of the mean separation) for all simulations - even for $\Lambda \mathrm{CDM}$ models where a higher virialization overdensity ( $\simeq 300$; cf. Kitayama \& Suto 1996, Appendix A) is expected. Results are rather insensitive to the choice of the linking length. This is not surprising because with lower linking lengths the algorithm finds substructures inside groups found with larger linking length. The most prominent of these substructures, however, are centered on the clusters themselves. Since we are looking only for the most massive groups in the simulation we will pick up the same centers almost independent of the linking length. This is also the reason why the results do not depend on the normalisation of the power spectrum as mentioned above. Note that the situation is different from that in finding galaxy-size halos where the results are sensitive to the galaxy finding algorithm (e.g. Klypin et al. 1997).

Fig. 2 shows that both the SCDM and OCDM (with $\Omega=0.35$ ) predictions lie well below the observed power spectrum whereas CHDM, $\Lambda$ CDM and BSI and $\tau$ CDM are in better agreement. Note, however, that on scales smaller than the scale of the maximum (about $120 h^{-1} \mathrm{Mpc}$ ) the observed power spectrum seems to be much steeper than all the simulated ones. From our data and the simulation we cannot conclude whether the peak in the power spectrum is real or just a statistical fluctuation. A similar structure of excess power has been found by several authors (e. g., Landy et al. 1996, Einasto et al. 1997b). However, the turnover at $k \approx 0.05 h \mathrm{Mpc}^{-1}$ seems to be real.

\section{Higher order statistics}

Besides the first order statistics (like the power spectrum or the correlation function) higher order statistics have been widely applied to cosmological problems. Mecke et al. (1994) have introduced Minkowski functionals into cosmology. They include informations about all orders of correlation functions. Minkowski functionals measure the geometry and topology of bodies. However, in cosmology one has to deal with point distributions. In order to describe such a point distribution with Minkowski functionals Mecke et al. (1994) proposed to surround each point by a sphere and calculate the Minkowski functionals of the resulting body. With increasing radius of these spheres the Minkowski functionals of the resulting body changes. 

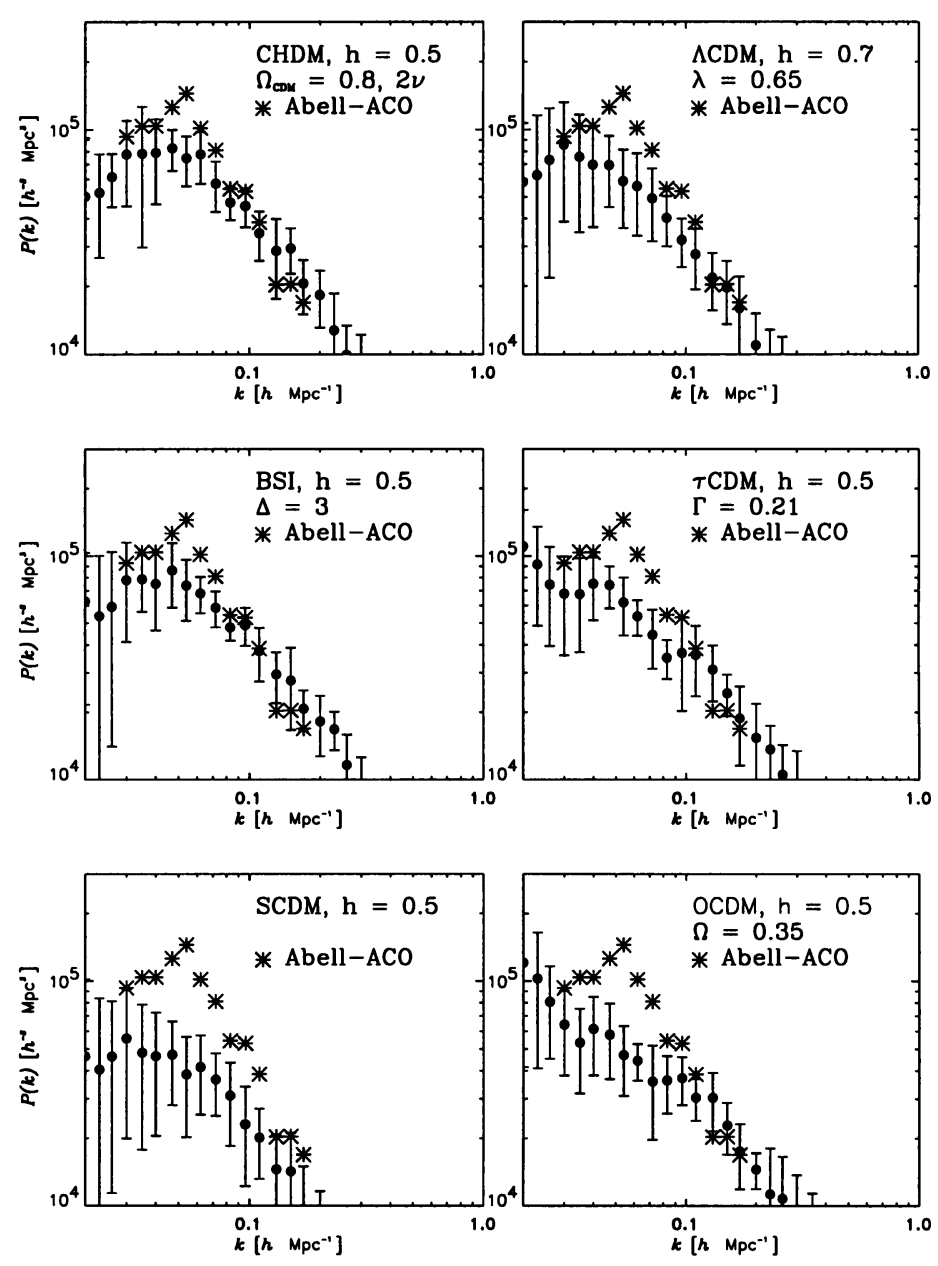

Figure 2. The cluster power spectrum of mock catalogs in different cosmological models in comparison with the spectrum of Abell/ACO clusters

Thus the radius can be used as diagnostic parameter in order to determine the differences between the point distributions under consideration.

In order to describe Minkowski functionals in an intuitive way let us consider a compact, convex three-dimensional body and the surrounding body at a distance $\varepsilon$. Then the volume of the surrounding body can be given in a series of $\varepsilon^{i}(i=0, \ldots, 3)$ where the coefficients are (with some constant factor) the four Minkowski functionals of this body. Therefore, the first and second Minkowski functionals are simply the volume and surface 
of this body. The first Minkowski functional is closely related to the void probability function. As the void statistics described by Gottlöber, Retzlaff, Turchaninov (1997) it is much less discriminative between models than the other functionals. The third functional is proportional to the integral mean curvature of the surface (in case of a sphere the radius) and the fourth is given by the mean Gaussian curvature and up to $4 \pi$ it is equal to the Euler characteristic. The Euler characteristic is closely related to the genus of isodensity surfaces which has become a commonly applied statistics in cosmology.

The sample of Abell/ACO clusters and the mock samples are threedimensional point distributions within the double cone which defines the sample geometry. It is clear that for small radii the resulting body is a set of disjoint spheres. With increasing radius these spheres start to overlap and build up more and more complicated structures with loops, voids and tunnels. Depending on the clustering properties the four Minkowski functionals show different behaviours. Obviously, in case of a highly clustered point distribution the first and second Minkowski functionals increase slower (total volume and total surface are smaller) than in the case of the Poisson distribution. The sign of the third Minkowski functional describes whether mainly convex (positive) or concave (negative) structures exist. Finally, the Euler characteristic exhibits the topology. For tunnels outweighing isolated objects and cavities it becomes negative. The Minkowski functionals for the Abell/ACO cluster sample and the set of mock catalogs of the four simulation samples have been calculated by Kerscher et al. (1997). The comparison of the Minkowski functionals of the observed and simulated samples has shown that the SCDM and TCDM models show significant departure from the observed cluster distribution whereas the $\Lambda$ CDM and BSI models fit reasonable well the data (for all functionals the differences were below the $1 \sigma$ level).

\section{Conclusions}

Comparing the observed and predicted distribution of clusters of galaxies we can discriminate between different cosmological models. Contrary to the case of galaxies the conclusions from numerical simulations of cluster distributions are independent of the cluster identification scheme.

Measuring the observed and simulated distributions of Abell/ACO clusters with the power spectrum, void statistics, and Minkowski functionals we found reasonable agreement of the observations with the BSI $(h=0.5$, $\left.\Delta=3, k_{b}^{-1}=1.5 h^{-1} \mathrm{Mpc}\right)$ and $\Lambda \mathrm{CDM}$ models $\left(h=0.7, \Omega_{C D M}=0.35\right)$. In case of the CHDM model $\left(h=0.5, \Omega_{C D M}=0.8,2 \nu\right)$ we have calculated only the power spectrum of mock catalogs. It fits reasonable well the data. 
Surprisingly, the $\tau$ CDM mock catalogs fit the data much worse than the BSI mock catalogs though the initial spectra for the simulations at $z=25$ are quite similar (cf. Fig. 1). The SCDM, OCDM $\left(\Omega_{t o t}=0.35\right)$ and TCDM $(n=0.9)$ models are clearly ruled out.

Note however, that none of these models can explain the apparent sharp break in the power spectrum at $k_{m} \approx 0.05 h \mathrm{Mpc}^{-1}$ and the steep slope with $n \approx-1.9$ at $k>k_{m}$.

SG acknowledges support from the Deutsche Akademie der Naturforscher Leopoldina with means of the Bundesministerium für Bildung und Forschung grant LPD 1996. JR acknowledges receipt of grant Go563/5-3 of the Deutsche Forschungsgemeinschaft. AK acknowledges receipt of NASA ATP grant.

\section{References}

Abell G.O., 1958, ApJ, 3, 211

Abell G.O., Corwin H.G., Olowin R.P., 1989, ApJS, 70, 1

Borgani S., Moscardini L., Plionis M., Górski K.M., Holtzman J., Klypin A., Primack J.R., Smith C.C., Stompor R., 1996, NewA 1, 321

Efstathiou G.P., Bond J.R., White S.D.M., 1992, MNRAS 258, 1P

Einasto J., Einasto M., Frisch P., Gottlöber S., Müller V., Saar V., Starobinsky A.A., Tago E., Tucker D., Andernach H., 1997a, MNRAS 289, 801.

Einasto J., Einasto M., Gottlöber S., Müller V., Saar V., Starobinsky A.A., Tago E., Tucker D., Andernach H., Frisch P., 1997b, Nature 385, 139

Gaztañaga E., Baugh C.M., 1997, astro-ph 9704246

Górski K.M., Hinshaw G., Banday A.J., Bennett C.L., Wright E.L., Kogut A., Smoot G.F., Lubin P., 1994, ApJ, 430, L89

Gottlöber S., Müller V., Starobinsky A.A., 1991, Phys. Rev., D43, 2510.

Gottlöber S., Mücket J.P., Starobinsky A.A., 1994, ApJ, 434, 417

Gottlöber S., Retzlaff J., Turchaninov V., 1997, Astrophysics Reports 2, 55.

Kerscher M., Schmalzing J., Retzlaff J., Borgani S., Buchert T., Gottlöber S., Müller V., Plionis M., Wagner H., 1997, MNRAS 284, 73.

Kitayama T., Suto Y., 1996, ApJ, 469, 480

Klypin A., Gottlöber S., Kravtsov A., 1997, astro-ph 9708191

Landy S.D., Shectman S.A., Lin H.L., Kirshner R.P., Oemler A., Tucker D.L., 1996, ApJ, 456, 1L

Mecke K., Buchert T., Wagner H., 1994, A\&A, 288, 697

Plionis M., Valdarnini R., 1991, MNRAS, 249, 46

Plionis M., Valdarnini R., 1995, MNRAS, 272, 869

Primack J., Holtzman J., Klypin A., Caldwell D., 1995, Phys. Rev. Lett. 74, 2160

Retzlaff J., Borgani S., Gottlöber S., Müller V., Plionis M., 1997, astro-ph 9709044

Stompor R., Górski K.M., Banday A.J., 1995, MNRAS, 277, 1225 